https://helda.helsinki.fi

\title{
Pustulose érosive du scalp
}

\section{Kluger, Nicolas}

2018-12

Kluger , N 2018 , ' Pustulose érosive du scalp ' , NPG Neurologie - Psychiatrie - Gériatrie , vol. 18 , no. 108 , pp. 333-335 . https://doi.org/10.1016/j.npg.2018.04.007

http://hdl.handle.net/10138/307725

https://doi.org/10.1016/j.npg.2018.04.007

publishedVersion

Downloaded from Helda, University of Helsinki institutional repository.

This is an electronic reprint of the original article.

This reprint may differ from the original in pagination and typographic detail.

Please cite the original version. 


\title{
CAS CLINIQUE
}

\section{Pustulose érosive du scalp}

\section{Erosive pustular dermatosis of the scalp}

\author{
N. Kluger
}

Dermatology, Allergology and Venereology, University of Helsinki and Helsinki University Central Hospital, Meilahdentie 2, PO Box 16000029 HUS, Helsinki, Finlande

Disponible sur Internet le 24 mai 2018

\section{MOTS CLÉS}

Alopécie ;

Pustulose érosive ;

Scalp ;

Sujet âgé ;

Zinc

\section{KEYWORDS}

Alopecia;

Elderly;

Erosive pustular

dermatosis;

Scalp;

Zinc
Résumé La pustulose érosive du scalp est une entité rare de cause inconnue touchant principalement les personnes âgées. Elle est souvent déclenchée par un traumatisme local et se présente sous la forme de pustules chroniques du cuir chevelu évoluant vers une alopécie cicatricielle. Son traitement est mal codifié. Nous rapportons le cas d'un homme de 80 ans qui a présenté une réponse spectaculaire après trois mois de traitement par une lotion de propionate de clobétasol associée à du gluconate de zinc par voie orale à $60 \mathrm{mg} /$ jour.

(c) 2018 Elsevier Masson SAS. Tous droits réservés.
Summary Erosive pustular dermatosis of the scalp is a rare entity of unknown cause, mainly affecting elderly people. It is often triggered by a local trauma, and features chronic pustules of the scalp evolving toward scarring alopecia. Its treatment is not well codified. We report on the case of an 80-year-old man who presented a dramatic response under topical clobetasol propionate and oral zinc gluconate at $60 \mathrm{mg} /$ day.

(c) 2018 Elsevier Masson SAS. All rights reserved.
La pustulose érosive du scalp (PES) est une dermatose chronique rare caractérisée par des pustules douloureuses stériles, des érosions et des croûtes du cuir chevelu [1].

Adresse e-mail : nicolas.kluger@hus.fi
Elle a été décrite à la fin des années soixante-dix par Burton [2] et Pye [3]. Elle touche principalement, mais pas exclusivement, les personnes âgées [4-6]. Les lésions s'étendent lentement au cours des mois ou des années qui suivent, entraînant une alopécie cicatricielle marquée $[5,6]$. L'évolution vers un carcinome épidermoïde local est 


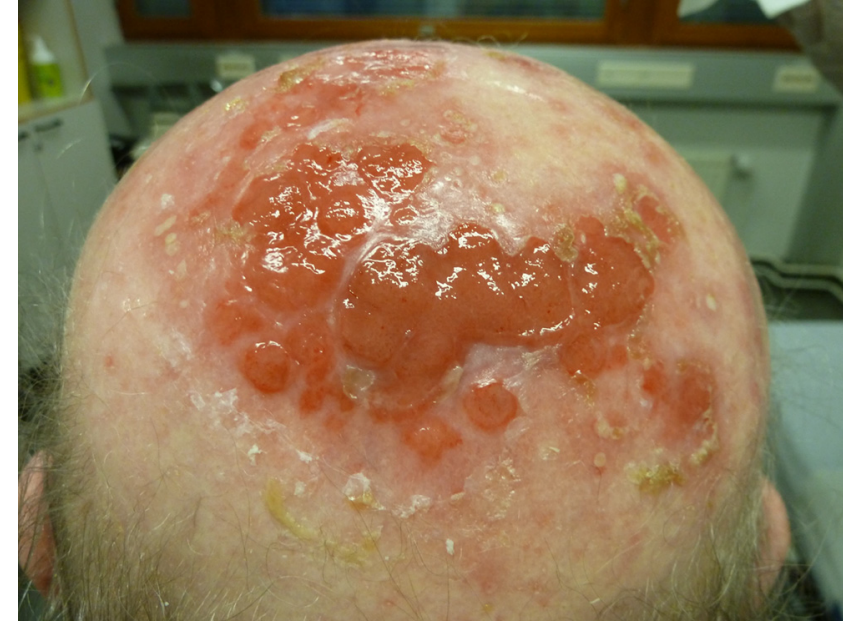

Figure 1. Plaques érosives et bourgeonnantes accompagnées de pustules et de croûtes jaunâtres en périphérie sur un scalp atrophique.

exceptionnelle [7] et probablement liée à des lésions préexistantes comme une kératose actinique. Cette maladie, assez mal connue, avec une évolution prolongée et de traitement difficile, est de fait sous-diagnostiquée avec un risque d'errance diagnostique [4] comme dans le cas que nous présentons ici.

\section{Cas clinique}

Un homme de 80 ans était adressé en consultation pour des lésions chroniques récidivantes du scalp évoluant depuis 7 mois. Il avait comme antécédents une hypertension artérielle, un diabète de type II, une goutte et une maladie de Bowen du scalp opérée en 2011.

L'histoire de la maladie avait débuté en juin 2017 par un traumatisme du scalp suite à une chute à domicile dans ses escaliers. Deux plaies, dont une large de $7,5 \times 3 \mathrm{~cm}$, était alors observées sur le scalp. Un traitement local associé à des antibiotiques (céphalosporines de $2^{\mathrm{e}}$ génération) était initié devant la découverte d'un staphylocoque doré méti-S sur les prélèvements. Le traitement antibiotique était répété à trois reprises entre juin et juillet 2017. Une biopsie réalisée fin juillet retrouvait essentiellement un tissu de granulation et un ulcère sans signe de malignité. Les soins locaux étaient continués jusqu'en novembre avec une bonne amélioration locale et une quasi-fermeture des plaies. Cependant, mi-novembre les lésions du cuir de chevelu récidivaient. Une nouvelle biopsie cutanée éliminait toute malignité locale et ne montrait qu'une réaction inflammatoire avec tissu de granulation.

À l'examen, en janvier 2018, le patient présentait une alopécie androgénétique. On retrouvait sur le sommet du cuir chevelu une large lésion ulcérée faite de tissu de granulation rouge et humide d'environ $7 \times 4 \mathrm{~cm}$, avec également de petites lésions satellites. On voyait également en périphérie des petites pustules et des croûtes jaunâtres (Fig. 1). Il n'existait pas d'impétigo ou de cellulite. Le reste de l'examen clinique était sans particularité. La peau du scalp était fine et atrophique.

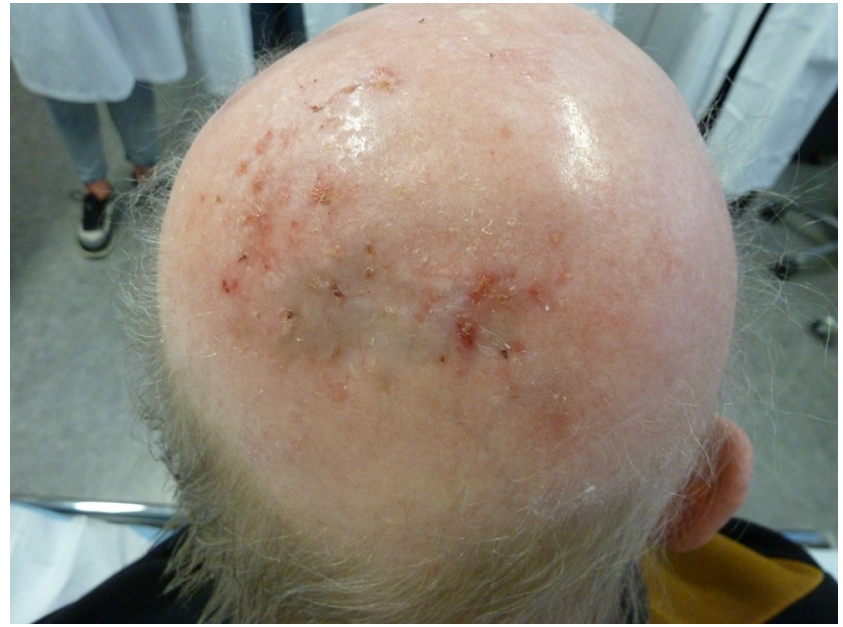

Figure 2. Évolution après 3 mois de traitement par gluconate de zinc $(60 \mathrm{mg} /$ jour) et application locale de propionate de clobétasol 3 fois par semaine.

L'histoire du patient (sujet âgé, traumatisme initial) et son tableau clinique (atteinte du scalp, lésions chroniques pustuleuses et érosives) correspondaient parfaitement au diagnostic de pustulose érosive du cuir chevelu. Un prélèvement bactériologique retrouvait à nouveau un staphylocoque doré. Le prélèvement mycologique était négatif. La CRP était discrètement élevée $(11 \mathrm{mg} / \mathrm{L})$ avec une hyperleucocytose $\left(11100 / \mathrm{mm}^{3}\right)$ et le taux de zinc était normal (11 micromol/L, N 9-18). Un traitement local par corticostéroïde de très forte activité (propionate de clobétasol) en solution était appliqué sur les lésions trois fois par semaine sous un pansement hydrocellulaire. Un traitement oral par gluconate de zinc $(30 \mathrm{mg} \times 2 /$ jour $)$ était associé pendant trois mois. Lors du contrôle à trois mois, les lésions étaient améliorées de façon spectaculaire (Fig. 2).

\section{Discussion}

Nous rapportons ici une histoire assez «typique » de pustulose érosive du scalp (PES) : un patient âgé avec une alopécie qui après un traumatisme " anodin » développe une réaction inflammatoire pustulo-croûteuse du scalp qui perdure plusieurs mois avec un traitement par antibiotiques et pansements locaux simples.

Dans la PES, une alopécie androgénétique sous-jacente et les lésions actiniques ne sont pas rares [5]. Divers facteurs déclenchants ont été rapportés. Une agression du cuir chevelu [4-6] est souvent retrouvée avant l'apparition des symptômes : traumatisme, contusions, brûlures locales ; infection herpétique ; traitement irritant local chimique (crème 5 -fluorouracile, trétinoïne topique), azote liquide ou après cryochirurgie, photothérapie dynamique, laser $\mathrm{CO}_{2}$, radiothérapie, chirurgie, greffes de peau ; exposition prolongée aux UV sur cuir chevelu gras ; et des taux diminués de zinc ont été soulignés [8]. Par ailleurs, diverses maladies auto-immunes ont été rapportées comme étant associées : polyarthrite rhumatoïde, thyroïdite de Hashimoto, hépatite auto-immune.

Cliniquement, la PES touche par définition le vertex. Le cuir chevelu est enflammé, avec des pustules jaunes, 
des croûtes jaunes ou hémorragiques, un cuir chevelu atrophique, et un amincissement des cheveux. La PES évolue lentement vers une alopécie cicatricielle progressive [5]. Une association avec une entité proche, la dermatose érosive pustuleuse des jambes (DEPJ), a été rapportée [5]. La DEPJ se caractérise par des érosions symétriques des jambes résultant de la confluence de pustules stériles survenant, le plus souvent, après un traumatisme minime chez des sujets âgés. Une carence en zinc a été rapportée chez des patients avec une DEPJ [9]. Pour certains, PES et DEPJ représentent une seule maladie [10].

Les résultats de laboratoire sont généralement sans particularité hormis un syndrome inflammatoire. Une carence en zinc ayant été rapportée [8], elle doit être recherchée au moins au moment du diagnostic. Les écouvillons bactériens et mycologiques locaux sont généralement négatifs, sauf si il existe une colonisation secondaire, avec un staphylocoque doré ou du Candida albicans principalement [4]. Les traitements anti-infectieux systématiques doivent être évités, car ils ne sont pas efficaces habituellement dans cette indication. Par contre, les écouvillons des pustules peuvent être répétés si nécessaire et un traitement d'épreuve discuté en cas de grandes pustules, de récurrences ou de résistance au traitement.

L'analyse microscopique d'une biopsie cutanée montre des lésions non spécifiques qui sont fonction du type de lésions échantillonnées (pustule, érosion, cicatrice) et de l'ancienneté de la maladie. À un stade précoce, l'histologie montre une inflammation chronique du derme supérieur avec un infiltrat mixte focal ou diffus composé de lymphocytes, de neutrophiles et de plasmocytes. La densité des cheveux est normale avec un nombre accru de follicules en phase catagène. À un stade plus évolué, on observe une fibrose du derme, une absence de glandes sébacées, une atrophie et une diminution du nombre (voire une absence complète) des follicules pileux. L'immunofluorescence directe est négative et élimine une pemphigoïde bulleuse [5].

Le traitement de la PES n'est pas codifié, et différents protocoles ont été essayés avec plus ou moins de succès. Les antibiotiques locaux et oraux ainsi que les désinfectants ne sont pas efficaces en l'absence d'une cause infectieuse. Les corticostéroïdes locaux à forte action anti-inflammatoire (propionate de clobétasol) sont efficaces. L'efficacité est évaluée cliniquement sur la disparition de l'inflammation, des érosions et des croûtes. Cependant, les dermocorticostéroïdes doivent le plus souvent être appliqués pendant longtemps avant une efficacité évidente (6 mois). Les rechutes se produisent au cours de l'année suivant l'arrêt du traitement, en moyenne après une période de trois mois. Par ailleurs, l'atrophie cutanée peut être aggravée par les corticostéroïdes. L'efficacité du tacrolimus à $0,1 \%$ une ou deux fois par jour, du calcipotriol à 0,005\% par jour et du gel de dapsone à $5 \%$ deux fois par jour a été rapportée dans des cas anecdotiques [6]. Le tacrolimus peut être utilisé en première ou seconde ligne de traitement en cas d'échec des corticostéroïdes. Son efficacité est évaluée après 3 mois [5]. Il peut également être utilisé comme traitement d'entretien pour prévenir les récidives deux fois par semaine [5]. En raison du risque de carcinome épidermoïde, le suivi du patient est recommandé. L'efficacité du zinc par voie orale a été soulignée dans certains cas, même en l'absence de carence en zinc $[8,11]$. L'association initiale zinc oral (gluconate de zinc) et dermocorticoïdes locaux suivie par le zinc seul pourrait être efficace. L'isotrétinoïne orale peut entraîner une amélioration durant quelques mois [12], mais l'atrophie cutanée peut être un facteur limitant pour un traitement de première ligne. La dapsone orale ne semble pas efficace selon les quelques données de la littérature [6]. Des conseils supplémentaires peuvent être donnés aux patients tels que la protection solaire et d'éviter les agressions supplémentaires du cuir chevelu : sèche-cheveux trop chaud, coiffage excessif, teinture, permanente ou blanchiment des cheveux [5].

\section{Conclusion}

La pustulose érosive du scalp est une pathologie rare qui survient principalement chez les sujets âgés, en général après un traumatisme du cuir chevelu. Son diagnostic est à connaître et sa prise en charge repose principalement sur les corticoïdes locaux associés à un traitement oral par zinc.

\section{Déclaration de liens d'intérêts}

L'auteur déclare ne pas avoir de liens d'intérêts.

\section{Références}

[1] Kluger N. Erosive pustular dermatosis of the scalp. Forum Nord Derm Ven 2017;22(2):38-9.

[2] Burton JL. Case for diagnosis. Pustular dermatosis of scalp. $\mathrm{Br}$ J Dermatol 1977;97:67-9 [Suppl 15].

[3] Pye RJ, Peachey RD, Burton JL. Erosive pustular dermatosis of the scalp. Br J Dermatol 1979;100(5):559-66.

[4] Caputo R, Veraldi S. Erosive pustular dermatosis of the scalp. J Am Acad Dermatol 1993;28(1):96-8.

[5] Starace M, Loi C, Bruni F, et al. Erosive pustular dermatosis of the scalp: clinical, trichoscopic, and histopathologic features of 20 cases. J Am Acad Dermatol 2017;76(6):1109-14.

[6] Broussard KC, Berger TM, Rosenblum M, et al. Erosive pustular dermatosis of the scalp: a review with a focus on dapsone therapy. J Am Acad Dermatol 2012;66(4):680-6.

[7] Lovell CR, Harman RR, Bradfield JW. Cutaneous carcinoma arising in erosive pustular dermatosis of the scalp. $\mathrm{Br} \mathrm{J}$ Dermatol 1980;103(3):325-8.

[8] Ikeda M, Arata J, Isaka H. Erosive pustular dermatosis of the scalp successfully treated with oral zinc sulphate. $\mathrm{Br} \mathrm{J}$ Dermatol 1982;106(6):742-3.

[9] Salavert M, Franck F, Amarger S, et al. Dermatose pustuleuse érosive des jambes : rôle de la carence en zinc ? Ann Dermatol Venereol 2006;133(12):975-8.

[10] Patton D, Lynch PJ, Fung MA, et al. Chronic atrophic erosive dermatosis of the scalp and extremities: a recharacterization of erosive pustular dermatosis. J Am Acad Dermatol 2007;57(3):421-7.

[11] El Kabbaj N, Dereure O, Guillot B. Pustulose érosive du cuir chevelu : 3 cas. Ann Dermatol Venereol 2005;132(5): 475-7.

[12] Petersen BO, Bygum A. Erosive pustular dermatosis of the scalp: a case treated successfully with isotretinoin. Acta Derm Venereol 2008;88(3):300-1. 